\title{
Assessment of Stress, Fear, Anxiety and Depression on COVID-19 Outbreak among Adults in South-Western Ethiopia
}

\author{
Kogila Palanimuthu', Hamba Yigazu², Eshetu Fikadu ${ }^{3}$, \\ Gemechu Gelalcha ${ }^{4}$, Yirgalem Bekele ${ }^{5}$, Getachew Birhanu', Birhanu Gutu ${ }^{7}$ \\ ${ }^{1}$ Assistant Professor, Department of Nursing, College of Medicine and health sciences, Dambi Dollo University, \\ Oromia, Ethiopia, ${ }^{2}$ Lecturer, Department of Public Health, College of Medicine and health sciences, Dambi Dollo \\ University, Oromia, Ethiopia, ${ }^{3}$ Graduate Assistant, Department of Public Health, College of Medicine and health \\ sciences, Dambi Dollo University, Oromia, Ethiopia, ${ }^{4}$ Graduate Assistant, Department of Psychology, School \\ of Education and Behavioral Sciences, Dambi Dollo University, Oromia, Ethiopia, ${ }^{5}$ Lecturer, Department of \\ Psychology, School of Education and Behavioral Sciences, Dambi Dollo University, Oromia, Ethiopia, ${ }^{6}$ Graduate \\ Assistant, Department of Public Health, Dambi Dollo University, Oromia, Ethiopia, ${ }^{7}$ Lecturer, Department of \\ Public Health, College of Medicine and health sciences, Dambi Dollo University, Oromia, Ethiopia.
}

\begin{abstract}
A study was conducted to assess the level of stress, fear, anxiety and depression among adults in residing in selected area. The objectives were to assess the socio demographic characteristics of adults, to find out the association between level of Stress, fear, anxiety and depression of the respondents with selected demographic variables.

A community based cross sectional study was conducted with 634 adults were selected by the multistage random sampling technique. The data were collected by using standardized questionnaires, fear on COVID 19 and Stress, anxiety and depression on COVID 19. The collected data was tabulated and analyzed. Descriptive and inferential statistics were used.

Researchers observed that $35.5 \%, 4.1 \%, 13.2 \%, 8.2 \%$ of the respondents reported moderate levels of fear, stress, anxiety and depression respectively and $21.3 \%, 1.1 \%, 1.7 \%, 1.3 \%$ of the respondents reported extreme levels of fear, stress, anxiety and depression respectively.It is essential and in evitable to alleviate the stress, fear, anxiety and depression among general population and provide positive metal health.
\end{abstract}

Keywords: Anxiety, COVID-19, Depression, Fear, Stress, South- Western Ethiopia.

\section{Introduction}

Till date many studies were conducted on COVID-19 like knowledge, attitude, practice, studies

\section{Corresponding Author:}

\section{Kogila Palanimuthu}

Assistant Professor, Department of Nursing, College of Medicine and Health Sciences, Dambi Dollo University (Government of Ethiopia), KellemWollega Zone,

Oromia, Ethiopia-260

e-mail: kogila@dadu.edu.et

Phone:+251946266424

Whats app Number: 09791868988 but psychological aspects of COVID-19 studies are very less in numbers. Therefore, this study is aimed to assess the level of Stress, fear, anxiety and depression (SFAD) status of our community on COVID-19 to help the local and national planners to identify the gap in public response to develop a protocol to overcome the SFAD from pandemic outbreak ${ }^{1}$.

One characteristic nature of infectious disease compared with other conditions is fear. Fear is directly associated with its transmission rate and medium (rapidly and invisibly) as well as its morbidity and mortality. This further leads to other psychosocial challenges including stigmatization, discrimination, and $\operatorname{loss}^{2}$. 
Morbidity and mortality statistics of Corona virus in 52 African countries are 13,600 and more infected cases, 742 deaths, recovered 2, 3583-6. Many of the ill people had contact with a seafood and animal market in Wuhan, a large city in eastern China, though it has since become clear that the virus can spread from person to person ${ }^{7-10}$.

An individual's personality characteristics, genetics, and childhood experiences with major stressors and traumas may also dictate their response to stressors. Acute Respiratory Syndrome (SARS) and Middle East respiratory syndrome (MERS) coronaviruses. Susceptibility seems to be associated with age and other health conditions ${ }^{8}$. COVID-19 has now been declared as a Public Health Emergency of International Concern by the $\mathrm{WHO}^{11,12}$.

In a cross-sectional study, Wang etal(2020) evaluated psychological impacts, depression, stress, and anxiety at the beginning of the COVID-19 outbreak. In this study, 1210 participants from 194 cities in China answered an online questionnaire. The author showed that $53.8 \%$ of these people experienced severe psychological impacts of the outbreak. Moreover, $16.5 \%, 28.8 \%$, and $8.1 \%$ of the respondents reported moderate to severe levels of depression, anxiety, and stress, respectively ${ }^{13}$.

\section{Objectives:}

General Objective: The aim of this study is to assess the level of Stress, fear, anxiety and depression on COVID-19 in Kelem Wollega Zone, Oromia, 2020.

\section{Specific Objectives:}

- To assess the respondents socio-demographic characteristics

- To determine the relationship between level of Stress, fear, anxiety and depression of the respondents with their selected demographic variables.

- To correlate the level of Stress, fear, anxiety and depression of the urban and rural respondents with their selected demographic variables.

\section{Materials and Method}

Non-experimental evaluative approach was used. A community based cross sectional study design was adopted. The source population was all the adults living in Kelem Wollega Zone during the study period. Adults who satisfying the inclusion criteria such as age group between 21-50 years with a multistage random sampling technique participants were chosen for the study during the study period.

Sample Size Determination: The required sample size was calculated by using single population proportion formula and open source Epidemiologic statistics for Public health(version 3.01) assuming that $50 \%$ of the community is having Stress, fear, anxiety and depression (SFAD) on COVID-19.

$$
\mathrm{n}=\frac{(Z \alpha / 2)^{2} \mathrm{p}(1-\mathrm{p})}{\mathrm{d}^{2}}
$$

where; $\mathrm{n}=$ sample size

$\mathrm{P}=$ by taking the calculated prevalence rate of $50 \%$, $\mathrm{Z} \alpha / 2=95 \%$ confidence level which is (1.96), $d=5 \%$ marginal error, Sample size as per openepi and formula results as 384 , Design effect is $1.5(384 \times 1.5=576)$ and after adding 10\% none response, final sample size 634 .

Sampling Procedure: In the first stage, three out of 12 woredas ( $25 \%$ of the total area) was selected by simple random sampling technique. In the second stage kebeles was selected from the woredas again using simple random sampling technique. In the third stage, a total of 634 households were selected using a systematic random sampling method. In this process sample was proportionally allocated to each selected kebele. Total number of households were obtained from the respective administrative areas and used to calculate the sampling fraction. Only one eligible individual was interviewed from the selected household. When two or more individuals are eligible in a household, only one was selected by lottery method.

Data collection instrument and Questionnaires development: The questionnaire was adapted from Structured standardized Five point likert Scale on Fear of COVID-19 (FCV 19S) and DASS Scale to assess the Depression, anxiety Stress on COVID-19 ( $2^{\text {nd }}$ edition Sydney psychology foundation) were used by Structured Interview Schedule. The questionnaire was translated into Afan Oromo and again translated back in to English, and comparisons was made on the consistency of the two versions.

Data Collection Procedure: Data collectors were trained on the objective of the study and techniques of interviewing the participants. Totally 15 diploma holders midwives who were working in the area was deployed as data collectors on each selected kebeles. Data collection was conducted face to face interview 
with structured questionnaire along with proper personal protective equipment. Respondent's doubts and queries also clarified by health workers. Supervisors followed the activities of data collectors daily and investigators guided the overall activities. Respondents were received pamphlets on coping tips to protect from covid-19 stress, fear, anxiety and depression.

Data Processing and Analysis: After the data was collected, filled questionaries' were checked manually for completeness and then coded. Entered and cleaned using Epidata version 3.11. Double entry made to minimize entry error and exported to SPSS version 23.0 for analysis. Descriptive statistics like frequency distribution, percentage, mean, standard deviation \& inferential statistics like ' $\mathrm{t}$ ' test and fried man test were used to analyze the data. P-Value $<0.05$ was considered to be declared statistically significant.

\section{Results}

The community based cross sectional study was carried out to assess the level of stress, fear, anxiety and depression on COVID-19 among study participants. The total of 634 participants participated in this study.

Socio demographic characteristics of the study participants were $238(37.5 \%)$ were belongs to $21-30$ years. $177(27.9 \%)$ rural and $457(72.1 \%)$ were from urban, in this $420(66.2 \%)$ participants were male and $214(33.8 \%)$ belongs to female.

Significant difference between male and female with respect to Factors of fear, stress, anxiety and depression on COVID19 among the respondents were presented in Table 1 and it is revealed that significance difference between male and female of respondents with regard tot he Factors of fear. Based on means core, the male respondents (1.8429) have high level of perception on fear than female respondents (1.6589).

Table: 1: t-test for significant difference between Male and Female with respect to Factors of fear, stress, anxiety and depression on COVID19 among the Respondents

\begin{tabular}{|l|c|c|c|c|c|c|}
\hline \multirow{2}{*}{$\begin{array}{l}\text { Factors of perception on } \\
\text { COVID19 }\end{array}$} & \multicolumn{4}{|c|}{ Gender } & \multirow{2}{*}{ t value } & \multirow{2}{*}{ P value } \\
\cline { 2 - 7 } & Mean & SD & Mean & SD & \\
\cline { 2 - 7 } & 1.8429 & 0.7841 & 1.6589 & 0.7382 & 2.849 & $0.005 *$ \\
\hline Fear & 1.2167 & 0.6008 & 1.2009 & 0.6293 & 0.307 & 0.759 \\
\hline Stress & 1.5690 & 0.9879 & 1.4720 & 0.9770 & 1.174 & 0.241 \\
\hline Anxiety & 1.4000 & 0.7889 & 1.3785 & 0.7701 & 0.327 & 0.744 \\
\hline Ovepression & 6.0286 & 3.1617 & 5.7103 & 3.1146 & 4.657 & $\begin{array}{c}<0.001 * * \\
<\mathbf{0 . 0 0 1} * *\end{array}$ \\
\hline
\end{tabular}

Fried man test for significant difference among mean ranks towards factors of fear, stress, anxiety and depression on COVID19 among the respondents represented in Table: 2 and it concluded that there is significant difference among mean ranks towards Factors of SFAD. Based on mean rank, Fear (2.79) is the most important factor, followed by anxiety (2.52), Stress (2.37) and Depression(2.13).

Table: 2: Fried man test for significant difference among mean ranks towards Factors of fear, stress, anxiety and depression on COVID19 among the respondents

\begin{tabular}{|c|c|c|c|}
\hline Factors of perception on COVID19 & Mean Rank & Chi-Square value & $P$ value \\
\hline Fear & 2.79 & \multirow{4}{*}{311.969} & \multirow{4}{*}{$<0.001 * *$} \\
\hline Stress & 2.37 & & \\
\hline Anxiety & 2.52 & & \\
\hline Depression & 2.13 & & \\
\hline
\end{tabular}


Figure 1-4 presents perception of fear on covid19 among 634 participants 274 (43.2\%) exhibits low level of fear and 135 (21.3\%) exhibits high level of fear and perception of stress on covid19, 49 (7.7\%) exhibits low level of stress and 07 (1.1\%) exhibits high level of stress.
Perception of anxiety on covid19, 50 (7.9\%) exhibits low level of anxiety and 26 (4.1\%) exhibits high level of anxiety and perception of depression on covid 19,93 (14.7\%) exhibits low level of depression and 07(1.1\%) exhibits high level of depression.

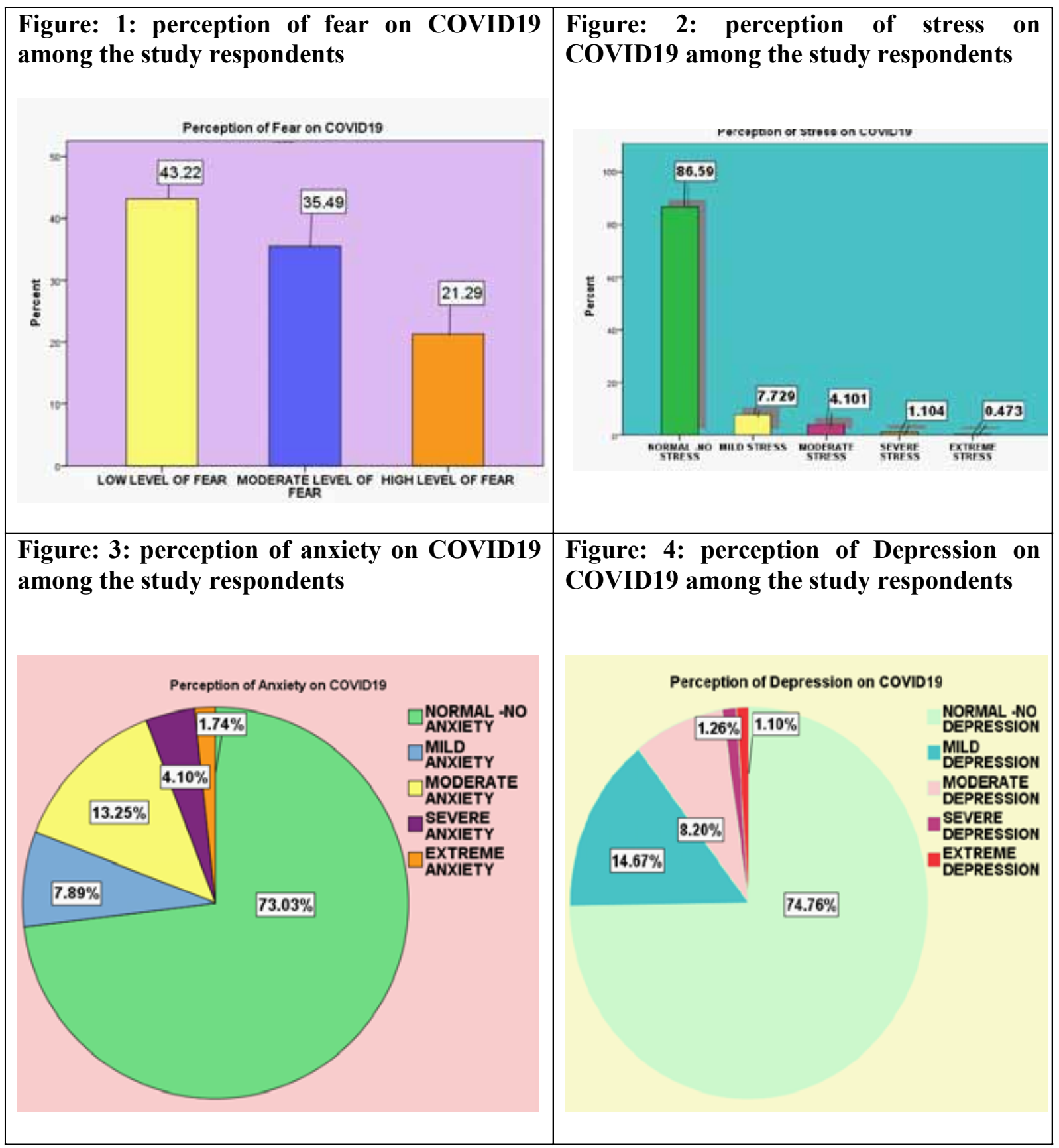

\section{Discussion}

Stress is a feeling of strain and pressure. It is a type of psychological pain. Small amounts of stress may be desired, beneficial, and even healthy. Positive stress helps improve athletic performance. It also plays a factor in motivation, adaptation, and reaction to the environment. Excessive amounts of stress, however, may lead to bodily harm. Stress can increase the risk of strokes, heart attacks, ulcers and mental illnesses. With the high levels Stress, fear, anxiety and depression (SFAD) of 
individuals may not think clearly and rationally when reacting to COVID-19. However, current treatment on COVID-19 worldwide has mainly focused on infection control, effective vaccine, and treatment cure rate (Dong et al. 2020; Wang et al. 2020).

The study was aimed to assess the stress, fear, anxiety and depression on COVID 19 on kellemwollega zone. It was supposed that, this study provided important information and created overall image on stress, fear, anxiety and depression on COVID 19 in kellemwollega zone.

In a cross-sectional study, Wang et al (2020) evaluated psychological impacts, depression, stress, and anxiety at the beginning of the COVID-19 outbreak, 1210participants from 194 cities in China answered an online questionnaire. The author showed that $53.8 \%$ of these people experienced severe psychological impacts of the outbreak. Moreover, $16.5 \%, 28.8 \%$, and $8.1 \%$ of the respondents reported moderate to severe levels of depression, anxiety and stress, respectively ${ }^{13}$.

On the other hand the researchers conducted the present study with 634 participants in different woredas $177(27.9 \%)$ rural and $457(72.1 \%)$ were from urban, in this $420(66.2 \%)$ participants were male and $214(33.8 \%)$ belongs to female and observed that $35.5 \%, 4.1 \%$, $13.2 \%, 8.2 \%$ of the respondents reported moderate levels of fear, stress, anxiety and depression respectively and also observed that $21.3 \%, 1.1 \%, 1.7 \%, 1.3 \%$ of the respondents reported extreme levels of fear, stress, anxiety and depression respectively. In present study 3 woredas were participated from that one woreda is exhibiting high level of fear on COVID-19 110 (33\%) comparing to all other woredas as follows: $10(6.7 \%), 15$ $(10 \%)$. These study results resembling the study results of other country.

\section{Conclusion}

Our study revealed that the majority of study participants had high level of fear, moderate level of stress and anxiety and very few showed that low level of depression. Researchers strongly recommending the Psychological first Aid training for health professionals and followed by psychological first aid to concern woreda respondents to help them to stabilize the mental health by reducing the fear on deadly pandemic disease COVID-19. It is essential and in evitable to alleviate the stress, fear, anxiety and depression among general population and provide positive metal health.
Acknowledgement: The authors wholeheartedly wish to thank Dr. Leta Tesfaye, President, Dr. Buli Yohanis Tasisa, Academic vice President, Prof. Yoseph Shiferaw Belayneh, Research and Community service vice President, Prof. Malkamu Tamiru, Research and Technology innovation Directorate Director, Dambi Dollo University, Oromia, Ethiopia for their support for completing the activities in a timely manner.

\section{Conflict of Interest: Nil}

Source of Funding: The Corresponding Author received funding from the Dambi Dollo University for conducting the research in 3 woredas and also obtained Permission for authorship and Publication of this article

Ethical Clearance: Official ethical clearance Letter was received from Research and Technology Innovative directorate office, Dambi Dollo University and it was presented to Kelem Wollega health department. All the information regarding the study, Researchers contact information, participants rights were provided in the first page of questionnaire. Written consent was obtained from each participant. Confidentiality, anonymity and privacy were maintained by excluding the name and ID of study participants from the questionnaire. Autonomous were maintained for both recruited \& non recruited Participants by who are not willing to participate in the study was respected and they were not recruited in the study. All the enrolled participants were informed about the rights to withdraw from the study at any point of time. Justice was maintained by randomization to select the participants and veracity were maintained by truthfulness in each stage of the study.

\section{References}

1. WMHC. Wuhan Municipal Health and Health Commission's Briefing on the Current Pneumonia Epidemic Situation in Our City. 2020.

2. Li Q, Guan X, Wu P, Wang X, Zhou L, Tong Y, et al. Early transmission dynamics in Wuhan, China, of novel coronavirus-infected pneumonia. N Engl J Med. 2020.

3. Centers for Diseases Control and Prevention Coronavirus disease (COVID-19) outbreak (2020)

4. CDC. 2019 Novel coronavirus, Wuhan, China. 2020 .

5. UNICEF, Coronavirus disease, strategies to overcome the stress and fear (COVID-19), (2020). 
6. WHO. Novel Coronavirus, fear and stress-China. 2020 .

7. Zhu N, Zhang D, Wang W, Li X, Yang B, Song $\mathrm{J}$, et al. A novel coronavirus from patients with pneumonia in China, 2019. N Engl J Med. 2020.

8. WHO. Novel Coronavirus-Japan (ex-China). 2020.

9. Virological.org. Novel 2019 Coronavirus Genome 2020.

10. Fehr AR, Channappanavar R, Perlman S. Middle East respiratory syndrome: emergence of a pathogenic human coronavirus. Annu Rev Med. 2017;68:387-99.
11. WHO. Statement on the second meeting of the International Health Regulations (2005) Emergency Committee regarding the outbreak of novel coronavirus (2019-nCoV). 2020.

12. Weijie Guan et all (February 28,2020) 'Clinical Characteristics of Coronavirus Disease 2019 in China', The New England Journal of Medicine.

13. Wang C, Pan R, Wan X, Tan Y, Xu L, Ho CS, et al. Immediate psycho-logical responses and associated factors during the initial stage of the 2019 coronavirus disease (COVID-19) Epidemic among the general population in China. Int J Environ Res Public Health. 2020;17(5). 